\title{
CARP: Context-Aware Resource Provisioning for Multimedia over $4 G$ Wireless Networks
}

\author{
Navrati Saxena ${ }^{1}$, Abhishek Roy ${ }^{2}$, and Jitae Shin ${ }^{1}$ \\ ${ }^{1}$ School of Info. \& Comm. Eng. Sungkyunkwan University, Suwon, Korea \\ ${ }^{2}$ WiBro System Lab, Samsung Electronics, Suwon, South Korea \\ \{navrati,jtshin\}@ece.skku.ac.kr, abhishek.roy@samsung.com
}

\begin{abstract}
In this paper a context-aware resource provisioning framework is developed for multi-system, heterogeneous $4 G$ wireless networks. The framework envisions that each individual sub-network is fairly independent, and uses Bayesian learning scheme for capturing user's mobility profiles. The concept of Asymptotic Equipartition Property (AEP) helps to predict the most likely path-segments, that the mobile user (MU) is going to follow in near future, with very good accuracy. This helps in proactive resource management along the MU's future paths and locations providing the optimal resource (e.g., bandwidth) reservation. Simulation results on a synthetic wireless traces corroborate this high prediction success and demonstrate sufficient improvement in delay, blocking and throughput of ongoing wireless multimedia sessions.
\end{abstract}

Keywords: 4G Heterogeneous wireless networks, Bayesian learning, resource management, Information Theory, mobile multimedia, AEP.

\section{Introduction}

The concept of packet-based, next-generation $4 G$ wireless networks [2] lies in the co-existence of multiple cellular access networks. These individual cellular networks might possess significant differences in topology, coverage area and transmission range. However, by using multiple radio interfaces, future mobile nodes can seamlessly switch between these technologies, thus achieving the ultimate goal of global roaming. The $4 G$ network can thus be conceptually visualized as a collection of multiple independent access sub-networks, each of which has its own cellular layout and separate location tracking technique. "Context-awareness" is perhaps the key characteristic of these next-generation wireless networks and associated applications. Mobility and location information is the most important "context" in these networks, because the information needed by the vast majority of MUs depend strongly on their current or near future location.

In this paper we have developed a new Context-Aware Resource Provisioning scheme CARP across mobile-user's most frequent routes in heterogeneous $4 G$ cellular networks. Operating in symbolic space, CARP uses Bayesian algorithm to capture the movement profiles of individual MU across such networks. Although, in such systems, there exists a wide number of possible routes from a 
particular source to a destination, a MU usually follows his/her most likely paths. A similar analogy, dealing with the asymptotic equipartition property (AEP) [1] states that among all the long-range sequences consisting of random variables, there exists a fairly small typical set 11] which contains most of the probability mass and controls the average behavior of all such sequences. Using this concept, CARP captures the MU's typical path segments and reserves bandwidth along the typical paths. This helps in seamless transmission of ongoing real-time wireless communication with specific Quality of Service (QoS) guarantee. Using a priority-based service classification scheme CARP is also capable of guaranteeing significant improvement in blocking of real-time multimedia sessions.

Section 2 reviews the major existing works in location and resource management in $4 \mathrm{G}$ networks. A symbolic domain description of such networks is shown in Section 3 . The new concept of capturing MU's frequently used routes is developed in Section 4. An efficient resource reservation and admission control scheme based on this route formation is discussed in Section 5. Simulation results in Section [6 demonstrates the improvement of QoS in ongoing wireless sessions. Finally Section 7 concludes the paper.

\section{Related Works: Mobility in 4G Wireless Systems}

Given the large body of work on resource management in a single cellular access system (e.g., PCS), we survey only the major contributions related to our context. The guard channel policy [8] and fractional guard channel policy [9] determine the number of guard channels for hand-offs. The shadow cluster scheme 6 ] estimates future resource requirements in a collection of cells in which a mobile is likely to visit in the future. An efficient scheme for mobility-based predictive admission control is also proposed in 12 for single-system wireless networks.

Some recent work has reported on the problem of location and resource management in a multi-system environment. It is shown in [1] that an integrated location management strategy can significantly outperform an independent operation of each sub-system's location management algorithm. Similarly recent researches have pointed out a significant trend towards seamless inter-networking of wireless LANs and cellular networks [3]. The issues of network selection [10] in heterogeneous wireless networks are also under investigation. The problem of optimal location management [7] in heterogeneous wireless networks is also recently pointed out. However, the problem of resource reservation and management for guaranteed QoS in a generic multi-system environment is yet to be effectively addressed. This motivates the development of $C A R P$.

\section{Symbolic Representation of $4 \mathrm{G}$ Wireless Systems}

Figure 1shows an example of an integrated $4 G$ wireless network, comprising a collection of satellite, PCS and campus area (IEEE 802.11 WLAN and Bluetooth) based independent sub-networks. Clearly, the coverage area of each individual sub-system can be discontinuous. Accordingly, the set of sub-networks that can 


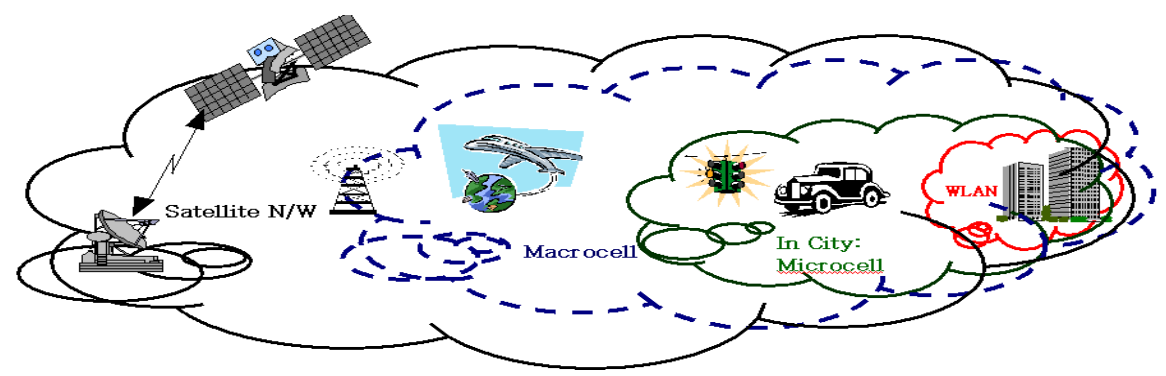

Fig. 1. A Multi-System Heterogeneous Wireless Network

be accessed concurrently by a mobile node is not constant but a function of its current location. Let, the $4 G$ network consists of $N$ sub-networks, where each sub-network is a collection of (either partitioned or overlapping) cells, such that $C_{i}^{j}$ represents the $j^{\text {th }}$ cell in the $i^{\text {th }}$ sub-network. If the mobile node is currently out of range of sub-network $S_{i}$, its location vector includes the cell $C_{i}^{\phi}$.

The movement pattern of the mobile node $(M N)$ can then be represented as a $N$-dimension random vector $\bar{X}$, where the $i^{\text {th }}$ element of the vector corresponds to the current cell of sub-network $S_{i}$. For instance, if $\bar{X}(2)=4$, the $M N$ is currently located in the $4^{\text {th }}$ cell of sub-network $S_{2}$. Characterizing the mobility as a probabilistic sequence suggests that it can be defined as a stochastic, vectorvalued process $\chi=\left\{\bar{X}_{n}\right\}$. where the "..." imply that the corresponding random variable can take any possible value within its range.

\section{Context-Awareness in Heterogenous Wireless Networks}

The MU's location uncertainty in heterogeneous wireless networks leads to uncertainty in the resources allocation and reservation policies. Once the location is managed properly, only then the network will be smart enough to reserve suitable wireless bandwidth. While the existing shadow cluster 6] approach provides the idea to preserve resources along the mobile's surrounding cells, it fails to give any generalized way to estimate these shadow clusters, and only assumes either some definite knowledge of user mobility or totally random users. The novelty of $C A R P$ lies in the fact that it not only captures the MU's locations, but also accumulates and predicts the user's typical routes. Thus, effectively $C A R P$ extends the concept of shadow cluster into heterogenous wireless networks to form a more smart resource management scheme.

\subsection{Probabilistic Estimation of User-Mobility}

$C A R P$ uses an online Bayesian Aggregation technique 4 to make the system knowledgeable enough about the MU's mobility profiles. Figure 2 provides a pseudo-code of our mobility prediction scheme. Each cell-vector $\bar{X}_{i}$ in the heterogenous wireless system is assigned to a weight $w_{i}$. Initially, all the weights are 


\section{Probabilistic Mobility Prediction}

1. initialize $m:=1$; assign weight $w_{i}:=1 / n$ to each instant $\bar{X}_{i}$;

3. repeat steps below until $\left(X_{p}-X_{a} \leq \varepsilon\right)$;

4. predict the next cell-vector (location) $\bar{X}_{p}:=\sum_{i=1}^{n} \frac{w_{i} \times \bar{X}_{i}}{w_{i}}$;

5. continue it's own movement; 6 . Capture the actual location $\bar{X}_{a}$;

7. compute the individual $\left(l_{i}\right)$ and overall $\left(l_{o}\right)$ prediction inaccuracy $l_{i}=-\left|\bar{X}_{a}\right| \ln \left|\bar{X}_{i}\right|-\left(1-\left|\bar{X}_{a}\right|\right) \ln \left(1-\left|\bar{X}_{i}\right|\right)$ and $l_{o}=\sum_{i=1}^{n} l_{i}$;

8. update the weights as: $w_{i}^{m+1}=w_{i}^{m} e^{-l_{i}}$;

Fig. 2. Bayesian Algorithm for Capturing User-Mobility

same, i.e., $w_{i}=1 / n$. The mobile-node now estimates it's own future-location by computing a weighted measure of these individual instances of cell-vectors (locations). Formally, the predicted location $\bar{X}_{p}$ is:

$$
\bar{X}_{p}=\frac{\sum_{i=1}^{n} w_{i} \times \bar{X}_{i}}{\sum_{i=1}^{n} w_{i}}
$$

The objective of $C A R P$ is to predict the future location correctly, so that the system becomes cognizant of user's mobility. If, $\bar{X}_{a}$ represents the actual location, the objective is to make the system cognizant enough so that $\bar{X}_{p}=\bar{X}_{a}$. The most fair measure of the prediction-inaccuracy (error) associated with this process is entropic loss 4]. This entropic loss $l_{i}$ for each location and its cumulative estimate in $m$ iterations $l_{i}^{c}$ is given by

$$
l_{i}=-\left|\bar{X}_{a}\right| \ln \left|\bar{X}_{i}\right|-\left(1-\left|\bar{X}_{a}\right|\right) \ln \left(1-\left|\bar{X}_{i}\right|\right), \text { and } l_{i}^{c}=\sum_{i=1}^{m} l_{i}
$$

At every iteration $m$, the weights associated with every time-instant $T_{i}$ are now updated as

$$
w_{i}^{m+1}=w_{i}^{m} e^{-l_{i}} .
$$

This procedure is iterated until $\bar{X}_{i}-\bar{X}_{a} \leq \varepsilon$, where $\varepsilon$ is a predefined precision. The effects of locations far from accuracy are reduced by exponentially decreasing their associated weights. In time the predicted location $\bar{X}_{i}$ approaches $\bar{X}_{a}$. The overall expected deviation form the optimality $\left(l_{o}\right)$ is given by:

$$
l_{o} \leq \min _{i} l_{i}^{c}+\ln (m)
$$

\subsection{Context-Aware Route Collection}

A close look into the life-style of a particular MU reveals that he/she typically follows only a small subset of all the paths, which essentially is guided by his life-style in the long run. The concept of typical set and asymptotic equipartition property (AEP) from information theory helps in obtaining this small set of highly probable routes maintained by a particular MU. 
Definition 1. The type [4] of a vector-sequence $\overline{\mathbf{x}}=\left\{\bar{x}_{1}, \bar{x}_{2}, \ldots, \bar{x}_{n}\right\}$ is the relative proportion of occurrences of each vector-symbol. Also, the set of all vectorsequences of a particular length and type is referred as the type class.

The essential power of the method of types arises from the fact that the number of types is always at-most polynomial [11. Hence, the crucial point to note here is that, there are polynomial number of types, but exponential number of sequences.

Result 1. If $\overline{\mathcal{V}}_{1}, \overline{\mathcal{V}}_{2}, \ldots, \overline{\mathcal{V}}_{n}$ denotes the set of vector-sequences drawn according to a specific distribution, then the probability of the sequence depends on relative difference between the type and the original distribution.

Hence, the type classes and the corresponding sequences that are far from the original distribution have exponentially smaller probabilities. This leads to the concept of typical set:

Definition 2. For a given $\epsilon>0$, a typical set $T_{Q}^{\epsilon}$ of sequences for the distribution $Q^{n}$ is defined as: $T_{\overline{\mathbf{v}}}^{\epsilon}=\{\overline{\mathbf{v}}: \mid$ Type - Distribution $\mid<\epsilon\}$.

All we now need is the probabilistic estimation of these typical sequences. Fortunately, this is exactly what the Shannon-McMillan-Brieman theorem [4]11] provides. According to this theorem, if $H(\chi)$ is the entropy of finite-valued stochastic ergodic process $\chi$, then

$$
-\frac{1}{n} \lg \left[p\left(\bar{V}_{0}, \bar{V}_{1}, \ldots, \bar{V}_{n-1}\right)\right] \rightarrow H(\chi)
$$

Equation 5 provides the basis of asymptotic equipartition property (AEP) [11] for any stationary, ergodic, stochastic process.

Result 2. AEP states that for a fixed $\epsilon>0$, as $n \rightarrow \infty, \operatorname{Pr}\left\{\chi \in T_{\overline{\mathrm{v}}}^{\epsilon}\right\} \rightarrow 1$.

This is basically similar to the weak law of large numbers [4 for ergodic, stochastic sequences, assuring that asymptotically almost all the probability mass of $\mathcal{V}$ is concentrated in the typical set. It encompasses the mobile user's most likely paths and determines the average nature of the large route-sequences.

\section{Advanced Resource Provisioning}

Resource reservation and admission control for multimedia services is a challenging research topic in the field of current and future generation wireless networks. The novelty of our approach lies in designing the bandwidth reservation scheme along the MU's typical (most probable) set of routes to ensure strict guarantee of QoS parameters like delay, throughput and blocking of real-time wireless applications; which had been missing from literature. The wireless audio-video applications are now capable of following the MU along his/her typical routes. Thus, the MU will be able to listen the news and watch a streaming video as he/she moves from one location to another. An estimation of reserved and free bandwidth in any heterogeneous cell $\bar{z}$ is determined by: 


$$
\begin{aligned}
B_{\text {res }}(\bar{z}, m) & =\operatorname{Pr}[\bar{z}] \times B_{m},=\sum_{\bar{X}^{n} \in T_{\epsilon}^{Q}}\left[\operatorname{Pr}\left[\bar{X}^{n}\right] \times \frac{f(\bar{z})}{\left|\bar{X}^{n}\right|}\right] \times B_{m}, \\
B_{\text {free }}(\bar{z}, m) & =B_{\text {tot }}-B_{\text {res }}(\bar{z}, m)
\end{aligned}
$$

where, $f(\bar{z})$ represents frequency of occurrence of $\bar{z}, B_{\text {tot }}, B_{m}, B_{\text {resv }}$ and $B_{\text {free }}$ are respectively the total bandwidth requirement of the mobile $m$, bandwidth requirement at $\bar{z}$, bandwidth reserved at $\bar{z}$ and free bandwidth at $\bar{z}$.

On arrival of a particular media packet in any $\bar{z}$, the bandwidth availability in that zone is checked for admission of the real-time video and voice stream. The unavailability of bandwidth leads to blocking of the media stream. On the other hand, if the bandwidth is available, the availability is checked only along the typical routes of the MU. The admission control of the multimedia packets is determined by the following condition:

$$
\begin{aligned}
& \sum_{\bar{z} \in \mathcal{V}^{n}} \operatorname{Pr}[\bar{z}] \times \beta\left(\bar{z}, B_{m}\right) \geq \tau \sum_{\bar{z} \in \mathcal{V}^{n}} \operatorname{Pr}[\bar{z}], \\
& \beta\left(\bar{z}, B_{m}\right)= \begin{cases}1, & B_{\text {free }}(\bar{z}) \geq B_{m} \\
\frac{B_{\text {free }}(\bar{z}, m)}{B_{m}}, & \text { otherwise }\end{cases}
\end{aligned}
$$

where $\tau$ is the admission threshold. Clearly, $\tau$ varies for voice, video and data packets. The data packets are given lower priority, and are admitted only if sufficient bandwidth is left (according to equations (6)-9) after admitting the real-time voice and video traffic.

\section{Simulation Results}

We have developed an object-oriented, discrete-event simulation environment for supporting MU's movements, associated prediction of typical routes and resource management for supporting location-aware multimedia services. The wireless voice services is modelled with exponentially distributed 'on' and 'off' periods (mean lengths $0.6 \mathrm{sec}$ and $0.8 \mathrm{sec}$ respectively). We have taken fluid queues having Weibullian inter-arrivals with heavy tails. The video sequences, are assumed to be encoded with ITU's (International Telecommunication Unit) H.264 [5] specifications with $176 \times 144$ pixel resolution and 7-30 frames/sec.

CARP reserves the highest bandwidth for ongoing voice (40\%-45\%-high priority) and the lowest for data traffic (10\%-15\%-low priority) along the MU's typical routes. Figure 3 demonstrates that the such a mobility-aware, proactive bandwidth reservation results in almost 50\%, 65\% and 30\% improvements respectively, in blocking of wireless voice, video and data streams, with increasing session arrival rates. In order to investigate into the mutual effect of the real-time voice and video streams over the other, we have looked into the throughput of voice and video traffic. Figure 4 shows that the with increasing session arrival rate the throughput of voice and video traffic lies around $65 \%-85 \%$ of the actual transmission data rate. Thus, we can conclude that such a location-aware 


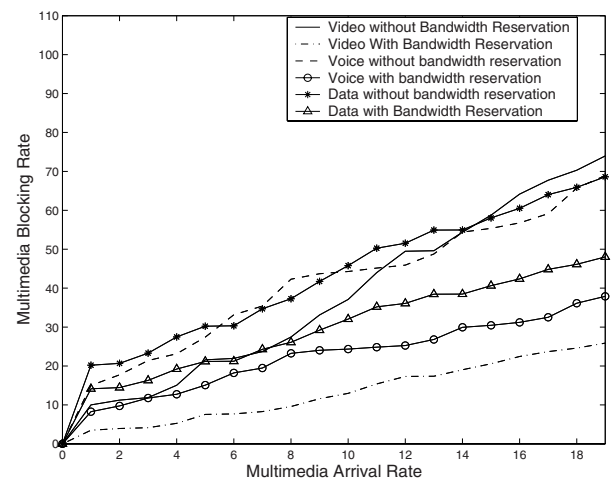

Fig. 3. Gain in Blocking of Mobile Multimedia

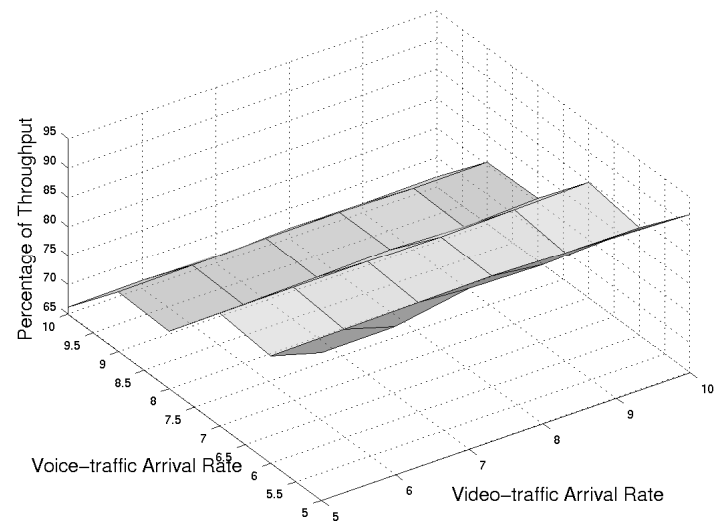

Fig. 4. Throughput of Voice and Video Traffic

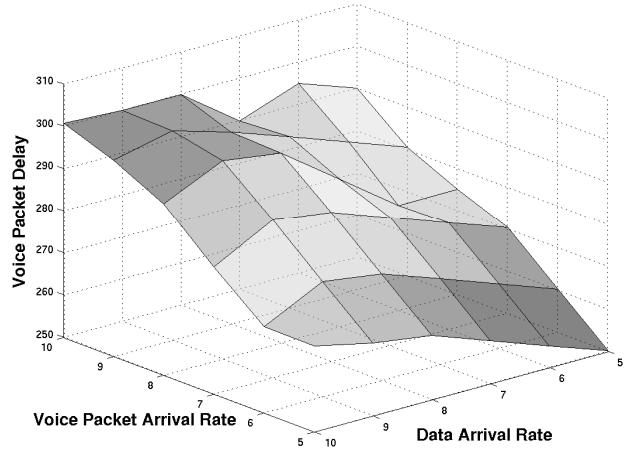

Fig. 5. Mutual Effects of Voice and Data Traffic

bandwidth reservation scheme has the potential for providing steady throughput of multimedia streams. Another point of concern is the mutual effects of traffic over the delay experienced by the MU in receiving the multimedia streams, while 
moving from one location to the another along the typical routes. Figure 5 shows that using this delay can be kept quite low for voice $(\approx 600 \mathrm{msec})$ services.

\section{Conclusions}

In this paper we have looked into the advanced resource reservation scheme for location-aware multimedia in heterogenous wireless networks. The developed system is quite cognizant about the MU's daily movement patterns and is capable of performing advanced reservation of wireless bandwidth along the MUs typical routes. This proposed framework aids in development of location-oriented multimedia with specific QoS (delay and throughput) guarantee, reduced blocking and seamless transmission along the MU's typical routes.

Acknowledgments. This work was supported by the Korea Research Foundation Grant funded by the Korean Government (MOEHRD)(KRF-2006-331D00358).

\section{References}

1. I. F. Akyildiz and W. Wang, "A Dynamic Location Management Scheme for NextGeneration Multitier PCS Systems", IEEE Transactions on Wireless Communications, vol. 1, no. 1, pp. 178-189, Jan 2002.

2. R. Berezdivin, R. Breinig and R. Topp, "Next-Generation Wireless Communications Concepts and Technologies", IEEE Comm. Mag., vol. 40, no. 3, March 2002.

3. D. Cavalcanti, D. Agarwal, C. Cordeiro, B. Xie and A. Kumar, "Issues in Integrating Cellular Networks, WLANS, and MANETS: A Futuristic Heterogeneous Wireless Network", IEEE Wireless Communications, pp. 30-41, 2005.

4. T. M. Cover and J. A. Thomas, Elements of Information Theory, John Wiley, '91.

5. ITU-T H.264; "Advanced video coding for generic audiovisual services", ITUT Specifications for Video Coding

6. D. A. Levine, I. F. Akyildiz and M. Nagshineh, "A resource estimation and call admission algorithm for wireless multimedia networks using the shadow cluster concept," IEEE Transactions on Networking, vol. 5, no. 1, pp. 1-12, February '97.

7. A. Misra, A. Roy and S. K. Das, "An Information Theoretic Approach for Optimal Location Tracking in Multi-System $4 G$ Wireless Networks", IEEE InfoCom, '04.

8. E. C. Posner and R. Guerin, Traffic Policies in Cellular Radio that Minimize Blocking of Handoff Calls, 11th Intl. Teletraffic Conference, Kyoto, Japan, Sept. 1985.

9. R. Ramjee, R. Nagarajan and Don Towsley, On Optimal Call Admission Control in Cellular Networks, Proceedings of IEEE INFOCOM, San Francisco, CA, 1996.

10. Q. Song and A. Jamalipour, "Network Selection in an Integrated Wireless LAN and UMTS Environment Using Mathematical Modeling and Computing Techniques", IEEE Wireless Communications, pp. 42 - 48, 2005.

11. A. Wyner and Z. Ziv, "Some Asymptotic Properties of the Entropy of a Stationary Ergodic Data Source with Applications to Data Compression", IEEE Transactions on Information Theory, vol. 35, no. 6, pp. 1250-1258, Nov. 1989.

12. F. Yu and V. Leung, "Mobility-based Predictive Call Admission Control and Bandwidth Reservation in Wireless Cellular Networks", Computer Networks, vol. 38. 